\title{
Heat transfer and solidification processes of alloy melt with undercooling_Part II: Solidification model
}

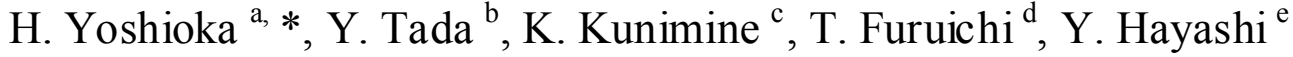 \\ a Department of Maritime Technology, Toyama National College of Maritime Technology, \\ 1-2 Ebie Neriya, Shinminato, Toyama 933-0293, JAPAN \\ ${ }^{\mathrm{b}}$ Department of Human and Mechanical Systems Engineering, Kanazawa University, \\ 2-40-20 Kodatsuno, Kanazawa, Ishikawa 920-8667, JAPAN \\ ${ }^{\mathrm{c}}$ Department of Mechanical Engineering, Akashi National College of Technology, 679-3 \\ Nishioka, Uozumi-cho, Akashi, Hyogo 674-8501, JAPAN \\ ${ }^{\mathrm{d}}$ Hitachi Cable, Ltd., 3-1-1 Sukegawa-cho, Hitachi, Ibaraki 317-0065, JAPAN

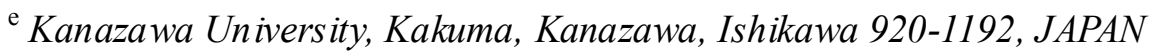

Keywords : Solidification; Undercooling; Metal \& Alloys; Crystal growth; Modeling; Heat transfer

Corresponding author:

Hideaki Yoshioka* (As sociate Professor)

Tel.: +81-766-86-5235; Fax: +81-766-86-5110.

E-mail: yoshi@toyama-cmt.ac.jp (H. Yoshioka) 


\begin{abstract}
The solidification process of undercooled alloy melts has been clarified experimentally in Part I of this paper. In this paper, using the experimental evidence, a solidification model linking macroscopic heat transfer and microscopic solidification is presented. The model reflects the microscopic solidification phenomena occurring until the thermodynamically unstable field shifts to equilibrium, consists of three fundamental processes: (1st stage) free growth, (2nd stage) crystal fattening with relaxation, and (3rd stage) equilibrium solidification. Based on this model, a numerical simulation is carried out for the temperature change, interface movement and solute concentration distribution during the solidification of undercooled Bi-Sn melt. Theoretical predictions of the temperature changes involving the recalescence, terminal time of the relaxation process and microsegregation for the solidified texture agree quantitatively with experimental observations.
\end{abstract}




\section{NOMENCLATURE}

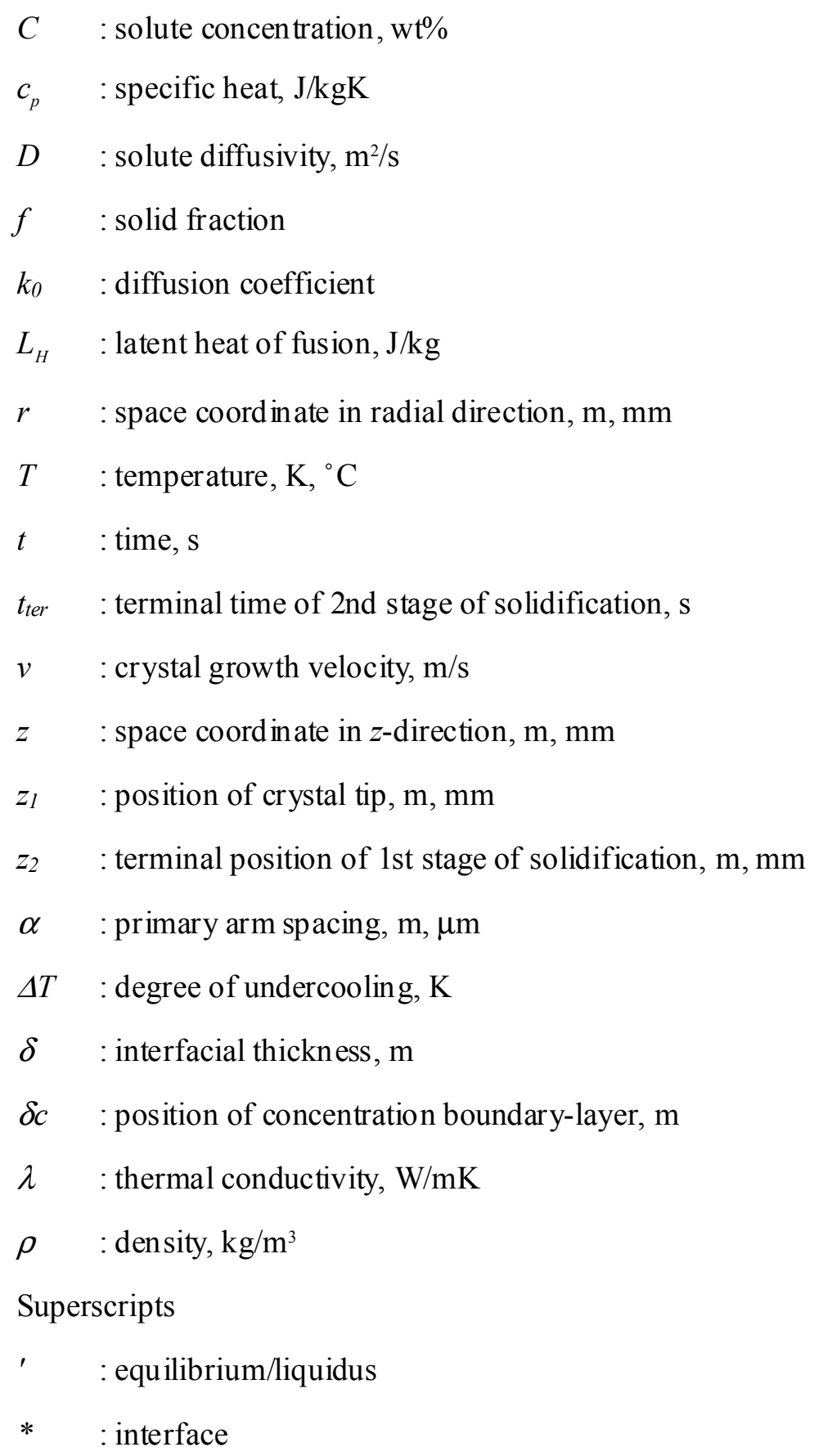


$i \quad$ : initial

$l, s, m$ : liquid, solid, mush 


\section{INTRODUCTION}

Solidification in metal and alloy processing produces solid phases which are a regular or dense assembly of atoms with the release of latent heat. To advance the solid front at an equilibrium/liquidus temperature, the sensible and latent heat should be removed by external cooling. During the initial solidification process, however, some undercooling is needed to drive the heterogeneous or homogen eous nucleation, and the undercooled metastable state sometimes appears as a liquid. This implies that the solidification process must take into account two types of heat extraction sources: initially distributed undercooling and external cooling. The initially distributed undercooling makes free crystal growth possible and also affects the subsequent solidification and heat transfer.

For free crystal growth in the undercooling state, various nonlinear models have been proposed since the theoretical work of Ivantsov [1] for pure metals. Typical solidification models for binary alloys were proposed by Trivedi et al. [2] and Lipton et al. [3-5]. These theories provide the temperature and concentration distributions around the crystal in a steady state, and are similar because they rely on a local equilibrium condition at the interface and make an assumption for determining the tip shape. Kabayashi [6] developed a numerical simulation using a phase-field model; in this simulation, the effects of noise and anisotropy on the dendrite shape are qualitatively analyzed. However, many other authors have exclusively considered the free growth in a uniform undercooling field. There are only a few works that deal with not only the free growth of numerous crystals in a non-uniform undercooling field but also the process after the free growth. In order to discuss the microscopic solidification phenomena from the viewpoint of controlling the microstructure, it is essential to develop a more realistic solidification model which considers the solidification process until the thermodynamically unstable field shifts to equilibrium. 
The authors explain the entire solidification process of undercooled alloy melts by macro- and microscopic experimental observations in Part I of this work [7]. In this paper (Part II), the theoretical kinetic link between macroscopic heat transfer and microscopic solidification behaviors is developed, based on the experimental evidence.

\section{SOLIDIFICATION MODEL}

As shown in Fig. 1, the heat transfer during solidification consists of the following basic phenomena: heat transfer until the undercooling state appears (1), recalescence due to the solidification driven by undercooling (2-1), relaxation of the non-equilibrium temperature and concentration fields under external cooling (2-2), and heat transfer with the release of latent heat over a range of temperatures (3). These macroscopic transport processes are represented in Fig. 1 in relation to the stages of microscopic solidification, which consists of free growth (1st stage), fattening of the crystal (2nd stage) and equilibrium solidification (3rd stage). A numerical simulation linking these processes was carried out.

\subsection{Modeling}

For the modeling, the physical coordinate systems are shown in Fig. 2(a), (b), which contrast the temperature field $T$ and solute concentration field $C$ with the crystal morphology. Here, $z$ is the space coordinate in the vertical direction to the cold wall (i.e., the distance from the cold wall), and $r$ is the space coordinate in the radial direction of the crystal in the cross section. $z$ ' in the $z$ direction is the position at which the field temperature at nucleation crosses the liquidus temperature at the initial concentration $T^{\prime}\left(C_{i}\right)$. Figure 2(a) shows the model for the non-equilibrium solidification process (1st and 2 nd stages). In the undercooled region formed near the cold wall $\left(0 \leq z \leq z^{\prime}\right)$, the 1st stage of solidification (free growth) begins. In this stage, the crystal grows in the $z$ direction with the temperature increase. Behind this is the 2nd stage of 
solidification (fattening), in which the crystal fattens with the temperature drop. The solute concentration $C$ and temperature $T$ fields in the $r$ direction, formed by the rejection of heat and solute from the solid/liquid interface, are shown in the lower two graphs of Fig. 2(a). Here, the negative temperature gradient or thermal undercooling, $T^{*}-T$, is the driving force for the rapid solidification during the 1 st stage. Although the thermal undercooling would be quickly reduced by the discharge of latent heat, the constitutional undercooling, $T^{\prime}-T^{*}$, must remain after the dissipation of the thermal undercooling because solute diffusivity is much less than thermal diffusivity. During the 2nd stage, the constitutional undercooling remaining between crystals may be relaxed gradually under the external cooling. Figure 2(b) shows the model for the equilibrium solidification process (3rd stage). During the 3rd stage, the solidification may proceed under thermodynamic equilibrium conditions, in which the liquid between crystals completely mixes.

To simplify the mathematical manipulation, it is as sumed that the mass of the side branches is included in the mass of the primary arm of the mass-equivalent interface, instead of in the mass of the actual dendritic interface. Furthermore, the following assumptions are made:

(1) The primary arm spacing is uniform.

(2) The lowering of the liquidus and solidus temperatures due to the curvature of the crystal surface is not considered.

(3) At the 1st stage, adiabatic crystal growth proceeds so that the temperature distribution of the liquid in front of the crystal tips does not change with time.

(4) Heat transfer due to the external cooling is conducted one-dimensionally in the $z$ direction.

(5) The mass transfer diffuses one-dimensionally in the $r$ direction, and no back-diffusion in the solid is considered.

(6) The effect of convection is not considered.

(7) The change of volume during solidification is not considered.

(8) The thermal properties do not depend on the temperature and solute concentration. The thermal properties in the mushy zone are weight-averaged according to the local solid fraction and change in the $z$ direction. 
Assumption (4) is supported by the reasoning that the boundary conditions at the cold wall and solidification front are restricted in the $z$ direction, and the arrangement of crystals is very dense. Furthermore, assumption (5) is supported by the reasoning that the local equilibrium conditions at the interface prevail along the $z$ direction, and the diffusion of solute, whose diffusivity is comparatively small, is governed only in the $r$ direction with a small diffusion length.

\subsection{Equations and calculation method}

The equations for the basic energy and mass balance of solute species (Eqs. (1), (3), (5)-(8) and (14)), and the initial condition (Eq. (2)) and the boundary conditions (Eqs. (4), (9)-(13)) corresponding to Fig. 2(a), (b), are represented in Table 1. In this table, subscripts $l, m$ and $s$ denote the liquid, mushy and solid regions, and the superscript* denotes the value at the interface. Equation (1) is the energy balance equation during the 1st stage. The left side of Eq. (1) represents the divergence of the latent heat of solidification. The first and second terms on the right side represent the change of enthalpy in the mushy zone due to recalescence and the change of the heat capacity due to the phase change, respectively. The location of the crystal tip $z_{1}$ is provided as

$$
z_{1}=\int_{t} v d t
$$

where $v$ is the velocity of the free growth, determined as a function of the initially distributed undercooling by the Lipton, Glicksman and Kurz (LGK) model [3,4]. In practice, a more complex treatment is required for the growth velocity close to equilibrium point $z$ ' because the LGK model cannot be applied for small undercooling. So, for simplicity, we assume that the growth velocity does not change after the fattening extends near the crystal tip. The terminal position of the 1st stage, $z_{2}$, is defined as the location where the existing thermal undercooling dissipates in the gap between the crystals, as determined by the following convergence condition: 
$T^{*}-T\left(\frac{\alpha}{2}\right)=10^{-6}$

Equations (5) and (6) in Table 1 are the mass balance equations in integral form. The first and second terms on the left side of Eq. (5) and (6) represent the mass of solute in the solid and the mass of solute in the liquid, respectively. The left side represents the mass of solute in the initial melt. The 1st stage of solidification is calculated for the mushy zone, which is divided into slab elements of width $\Delta z$ for the numerical simulation. For the first element, the interfacial temperature $T^{*}(\Delta z, t)$ is calculated by Eq. (1) for an assumed solidification rate $\Delta f$ in time increment $\Delta t$. If the obtained $\Delta f$ satisfies the mass balance determined in Eq. (5) or (6), the calculation may be carried out in succession for the next element, until the existing thermal undercooling diss ipates completely. In the calculation, the following relationship is used:

$\delta=\frac{\alpha \sqrt{f}}{2}$

where $\delta$ is the interfacial thickness, $\alpha$ is the primary arm spacing, and $f$ is the solid fraction. Additionally, to solve Eqs. (5) and (6) in Table 1 and (16) above, the temperature and concentration profiles within the bound ary layer must be known. These profiles in the $r$ direction are approximated by the secondary curve as follows:

$$
\begin{aligned}
& X(r)=a_{1} r^{2}+a_{2} r+a_{3} \quad\left(X=T, C_{l}\right), \\
& a_{1}=\frac{\xi}{2(\psi-\delta)}, \quad a_{2}=-2 \psi a_{1}, \quad a_{3}=X^{*}-a_{1} \delta^{2}-a_{2} \delta .
\end{aligned}
$$

In the case of the temperature distribution $(X=T)$,

$\xi=\frac{\rho L_{H}}{\lambda} \cdot \frac{d \delta}{d t}$ 
and in the case of the concentration distribution $\left(X=C_{l}\right)$,

$\xi=\frac{C^{*}\left(1-k_{0}\right)}{D} \cdot \frac{d \delta}{d t}$

where $C^{*}$ is equal to the liquidus concentration $C^{\prime}\left(T^{*}\right)$ because of assumption (2). If the boundary layer does not attain the center position between crystals, $\psi$ is the position of the boundary layer $\delta_{T}$ or $\delta_{C}$, or else $\psi$ is the position of half of the primary arm spacing, $\alpha / 2$. The boundary layer is expressed as

$\delta_{X}=\delta+\frac{2\left(X^{*}-X_{i}\right)}{\xi}$

The derivation of the above equations is described in Appendix A. For the concentration in the solid $C_{s}$, the value at the interface, which is determined by the solidus line, is fixed continually.

At the 2 nd stage, the calculation is carried out by coupling the en ergy equation (Eq. (3)) and the mass equation (Eq. (5) or (6)) using Eq. (17). Equation (3) is Fourier's differential equation, which includes a heat generation term due to the solidification. It is solved under the boundary condition for uniform heat flux at the cold wall, which is expressed as Eq. (4). Fourier's differential equation is calculated by the forward difference method with the conditions of $\Delta z=0.1$ $\mathrm{mm}, \Delta t=0.0001 \mathrm{~s}$. Moreover, the change in the solid fraction is calculated as satisfying Eq. (5) or (6) with the temperature recovery method [8]. The terminal time of the 2 nd stage $t_{\text {ter }}$ is defined as the time required for the constitutional undercooling to dissipate in the gap between crystals for the entire undercooled region $\left(0 \leq z \leq z^{\prime}\right)$, as determined by the following convergence condition:

$$
\frac{C_{l}^{*}-C_{l}\left(\frac{\alpha}{2}\right)}{C_{i}} \leq 10^{-6} .
$$


At the 3rd stage, the system is treated as a simple one-dimensional heat conduction problem, which can be solved by coupling the energy equations (Eq. (7) and (8)) and the mass equation (Eq. (14)). Equation (9) is the boundary condition at the cold wall, and Eq. (10) is the boundary condition at the adiabatic wall. Equations (11) and (12) are the boundary conditions for the liquid-mushy interface and mushy-solid interface, respectively, where $T^{\prime}$ is the liquidus temperature. A slight undercooling which remains at the crystal tips is ignored. Equation (13) describes the advance of the eutectic solid.

\section{SIMULATION RESULTS}

After the microscopic solidification process was linked to the macroscopic transport process, as shown in Fig. 1, the numerical simulation was carried out. The results predicted for $\mathrm{Sn}-30 \mathrm{wt} \% \mathrm{Bi}$ are shown in contrast with the experimental results in Fig. 4 and Fig. 5. In the calculation, the initial condition was set with the temperature at the solidification start. Also, we set the crystal spacing $\alpha=20 \mu \mathrm{m}$ and heat flux $q=-140 \mathrm{~kW} / \mathrm{m}^{2}$.

Figure 3 shows the change of the temperature distribution with time. From this, the series of thermal behaviors, which move from non-equilibrium conditions to equilibrium conditions, can be understood more clearly. In this figure, an initial undercooling state exists, as shown by the shaded region. First, the initial undercooling, which has a magnitude of about $17 \mathrm{~K}$ at the cold wall, collapses due to nucleation, and then the temperature rises due to the release of latent heat during the free growth. At each time of $0.35,1.35,3.86 \mathrm{~s}$, temperature rises (recalescence) occur up to $2.5,4.5,6.5 \mathrm{~mm}$, respectively, and this agrees with the experimental result. The magnitude of recalescence measured in the experiment appears less than the analytically predicted result. This is due to a thermocouple error in the experiment. After the recalescence, the temperature descends progressively from the cold wall by external cooling. The analytically predicted result 
generally agrees with the experimentally measured result, even if the boundary conditions are strictly different from the experimental conditions.

Figure 4 shows the movement of the solid/liquid interface corresponding to the temperature field described above. A crystal promoted by initially distributed undercooling grows in the $z$ direction. Next, fattening of the crystal appears. Also, a eutectic solid appears as the temperature drops below the eutectic point. During the free growth, as shown by the darker shaded region, the growth velocity decreases away from the cold wall, and the morphology thins down from the root to the tip. This response depends on the magnitude of the initial undercooling. Although the fattening front of the crystal keeps about a 1-mm difference with the tip of the crystal near the cold wall, as shown by the lighter shaded region, the difference shortens progressively away from the cold wall. Consequently, the time required for the crystal tip to reach the equilibrium point at $10.5 \mathrm{~mm}$ is $9.12 \mathrm{~s}$, and the terminal time of the relaxation process $t_{t e r}$, i.e., the time at which the crystal tip starts again, is $9.72 \mathrm{~s}$. This result matches the experimental observations that the free growth and fattening of the crystal do not always occur as separate stages in metallic alloy melts.

Figure 5 shows the solute concentration map for the solidified texture. The lines represent the solute concentration measured experimentally. The error bars were decided by the spatial resolution of the X-ray analyzer. At $z=10 \mathrm{~mm}$, a dendritic structure was observed, so the concentration along the centerline of the secondary arm is shown by the broken line. The solute concentration along the crystal center axis decreases in the undercooled region, and is a constant value in the region not undercooled. In the $r$ direction, the solute concentration decreases during the process of free growth and increases during the process of fattening of the crystal. Also, the eutectic concentration $C_{e}$ is formed between the crystals. In conclusion, it is shown that the model presented is able to describe the microstructure and microsegregation that appears during the complicated solidification process accompanied by undercooling. 


\section{CONCLUSIONS}

The solidification process of undercooled alloy melts was studied theoretically, and the following conclusions were obtained.

1. A good model linking macroscopic heat transfer and microscopic solidification has been presented. The microscopic model consists of three fundamental solidification processes: (1st stage) free growth with recalescence, (2nd stage) fattening of the crystal with the relaxation of constitutional undercooling, and (3rd stage) equilibrium solidification.

2. The relaxation processes of thermodynamically unstable temperature and concentration fields were clarified in relation to the solid/liquid interface morphology in the model.

3. Based on this model, by using the difference method, the temperature change, interface movement and microsegregation during solidification of undercooled metals can be calculated.

4. Theoretical predictions of the temperature changes involved the recalescence, terminal time of the relaxation process and final concentration distribution in the solid phase. These predictions agree quantitatively with the experimental observations.

\section{APPENDIX A. Derivation of the temperature $T$ and concentration $C_{l}$ profiles in the radial direction of a crystal, and the temperature and concentration boundary layers $\delta_{T}, \delta_{C}$}

For a transport quantity such as temperature or concentration, its profile $X$ can be approximated by the secondary curve

$$
X(r)=a_{1} r^{2}+a_{2} r+a_{3} \quad\left(X=T, C_{l}\right) .
$$

This satisfies the boundary conditions

at $r=\delta: \quad X=X^{*}$ 
at $r=\delta: \quad \frac{d X}{d r}=-\xi$

at $r=\delta_{X}: \quad X=X_{i}$

at $r=\delta_{X}: \quad \frac{d X}{d r}=0$,

where $\delta_{X}$ is the thermal or concentration boundary layer, $\delta_{T}$ or $\delta_{C}$, and $\xi$ is the rejection or diffusion of heat or solute at the interface, which is expressed as

$\xi=\frac{\rho L_{H}}{\lambda} \cdot \frac{d \delta}{d t}$ for the temperature field $(X=T)$

$\xi=\frac{C^{*}\left(1-k_{0}\right)}{D} \cdot \frac{d \delta}{d t}$ for the concentration field $\left(X=C_{l}\right)$.

The radial direction of a crystal is finite in polycrystal growth. Hence, when interference of the boundary layer occurs between neighboring crystals, the following boundary condition is used instead of Eqs. (A.4) and (A.5):

at $r=\alpha / 2: \quad \frac{d X}{d r}=0$

Rearranging Eqs. (A.2), (A.3) and (A.5) (or Eq. (A.8)) by Eq. (A.1) yields

$$
\left(\begin{array}{ccc}
2 \delta & 1 & 0 \\
\delta^{2} & \delta & 1 \\
2 \psi & 1 & 0
\end{array}\right)\left(\begin{array}{l}
a_{1} \\
a_{2} \\
a_{3}
\end{array}\right)=\left(\begin{array}{c}
-\xi \\
X^{*} \\
0
\end{array}\right),
$$

where $\psi$ is $\delta_{X}$ or $\alpha / 2$. By solving the above equation, we obtain 


$$
\left(\begin{array}{l}
a_{1} \\
a_{2} \\
a_{3}
\end{array}\right)=\left(\begin{array}{c}
\xi /\{2(\psi-\delta)\} \\
-2 \psi a_{1} \\
X^{*}-a_{1} \delta^{2}-a_{2} \delta
\end{array}\right) .
$$

Also, from Eq. (A.4)

$$
-\frac{\xi}{2}\left(\delta_{X}+\delta\right)+X^{*}=X_{i}
$$

Transforming this,

$$
\delta_{X}=\delta+\frac{2\left(X^{*}-X_{i}\right)}{\xi}
$$

\section{REFERENCES}

[1] Ivantsov GP, Dokl Akad Nauk USSR 1947;58:567.

[2] Trivedi R, Tiller WA, Acta Metall 1978;26:679.

[3] Lipton J, Glicksman ME, Kurz W. Mater Sci Eng 1984;65:57.

[4] Lipton J, Glicksman ME, Kurz W. Metall Trans 1987;18A:341.

[5] Lipton J, Kurz W, Trivedi R. Acta Metall 1987;35:957.

[6] Kobayashi R. Physica D 1993;63-3:410.

[7] Yoshioka H, Tada Y, Kunimine K, Furuichi T, Hayashi Y. Heat transfer and solidification processes of alloy melt with undercooling_Part I: Experiment results. Acta Mater 2005.

[8] Ohnaka I, Fukusako T. Trans ISIJ 1977;17:410. 


\section{Figure Captions}

Fig. 1. Link between the transport process and the solidification process.

Fig. 2. Physical coordinate systems: (a) non-equilibrium solidification (1st and 2nd stages); (b) equilibrium solidification (3rd stage).

Fig. 3. Comparison of the simulation result and experimental data on the time change of the temperature distribution for $\mathrm{Sn}-30 \mathrm{wt} \% \mathrm{Bi}$.

Fig. 4. Movement of the solid/liquid interface for $\mathrm{Sn}-30 \mathrm{wt} \% \mathrm{Bi}$.

Fig. 5. Solute concentration map of the solidified texture for $\mathrm{Sn}-30 \mathrm{wt} \% \mathrm{Bi}$.

\section{Table Caption}

Table 1

Equations of the basic energy and mass balance equations of solute species, and initial condition and boundary conditions for the non-equilibrium solidification process (1st and 2nd stages) and for the equilibrium solidification process (3rd stage). 
We are submitting our manuscript entitled "Heat Transfer and Solidification Processes of Alloy Melt with Undercooling-Part II: Solidification model." We would like to submit this paper to the Editor, Professor Richard Wagner, because this is one of two revised papers of the manuscript submitted previously (Ms No. A-05-93). We rewrote and revised the previous paper according to the reviewer's comments, split it into two separate papers and are resubmitting the two papers as individual submissions. This paper is the second of the two papers. In this paper, we have proposed a new model which links the microscopic solidification process and the macroscopic heat transfer conditions. We hope that this paper is suitable and acceptable for publication.

\author{
Hideaki Yoshioka \\ Toyama National College of Maritime Technology \\ Tel.: +81-766-86-5235; Fax: +81-766-86-5110. \\ E-mail: yoshi@toyama-cmt.ac.jp
}


Fig. 1

(1) Heat transfer

$\begin{aligned} & \text { - temperature distribution } \\ & \text { ( meta-stable undercooling })\end{aligned}$
$\bigvee$
(2-1) Recalescence

- temperature distribution

- concentration distribution

- - - - - - - - - - - - - - - -

(2-2) Relaxation /Recovery

- temperature distribution

- concentration distribution

(3) Heat transfer

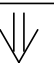

- temperature distribution

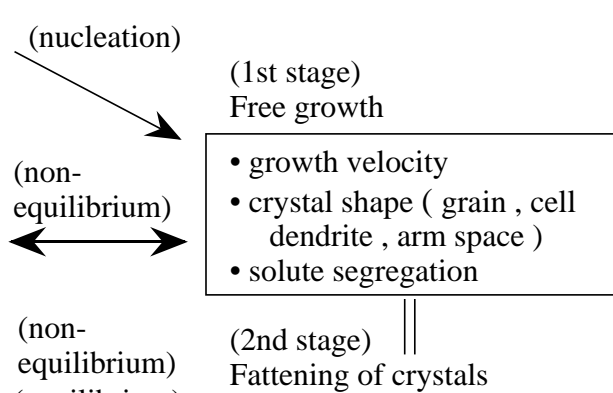

equilibrium) Fattening of crystals

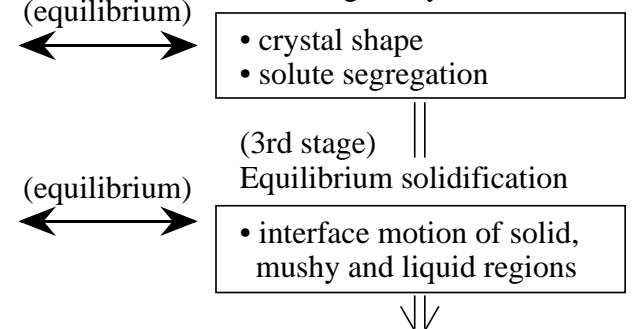

- solid with microstructure and microsegregation 
Fig. 2

(a)

$\underline{\text { direction }}$

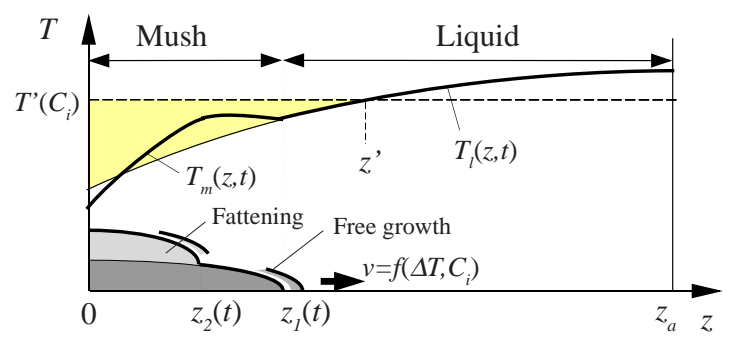

$\underline{r \text { direction }}$

C

C

$C_{i}$

$\bigvee_{C_{l}(r, t+\Delta t)}^{C_{l}(r, t)}$

$\begin{array}{lllll}0 & \delta(t) & \delta c(t) & \delta(t) \delta(t+\Delta t) & \alpha / 2\end{array}$

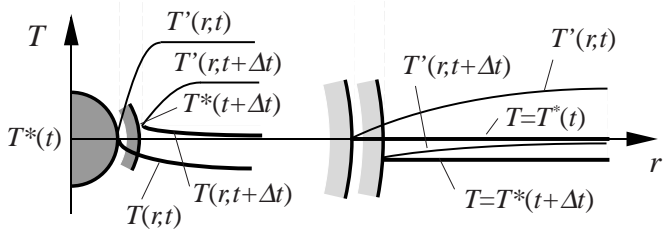

(1st stage)

Free growth/Recalescence (2nd stage)

Fattening of crystal/Relaxation (b)

$\underline{z \text { direction }}$

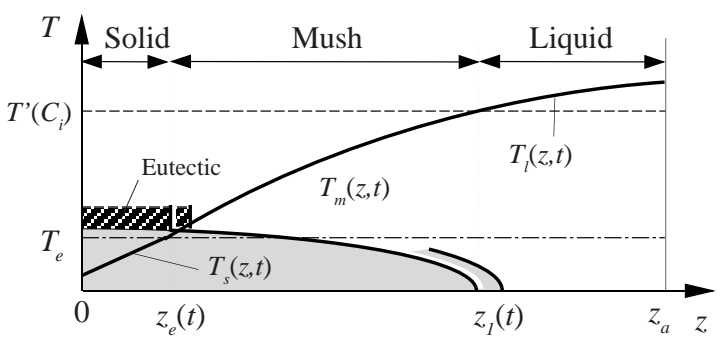

$\underline{r \text { direction }}$

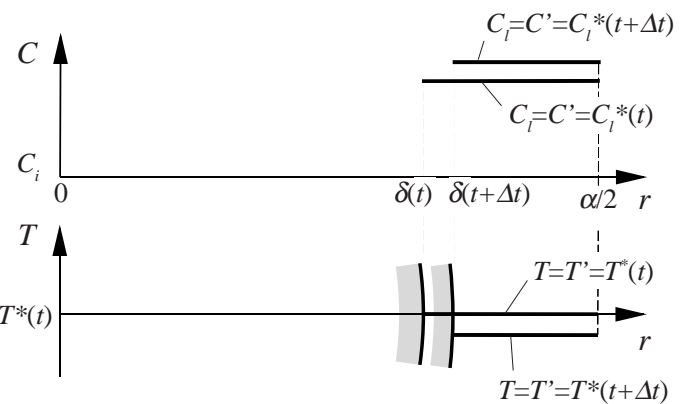

(3rd stage)

Equilibrium solidification 


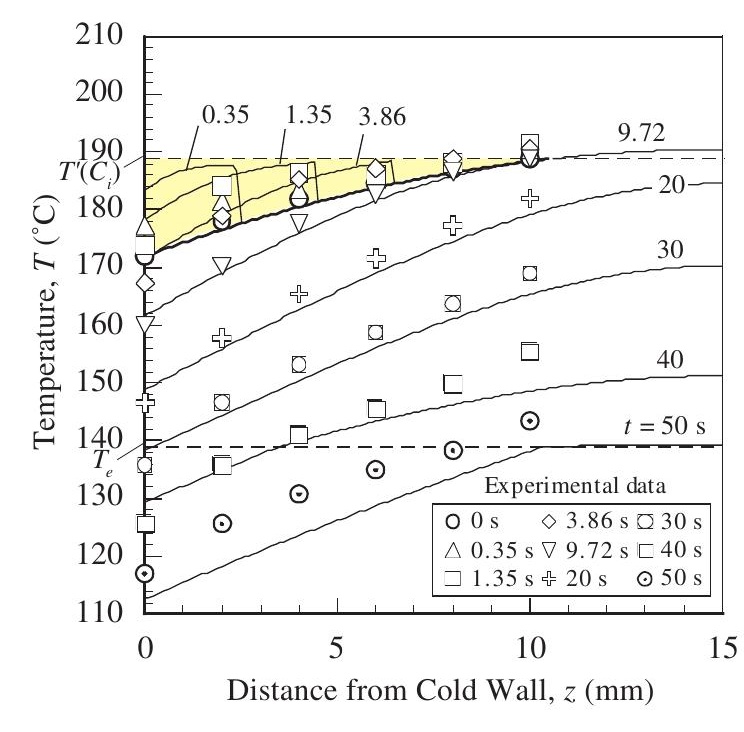

Fig. 3

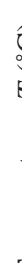

15

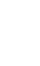




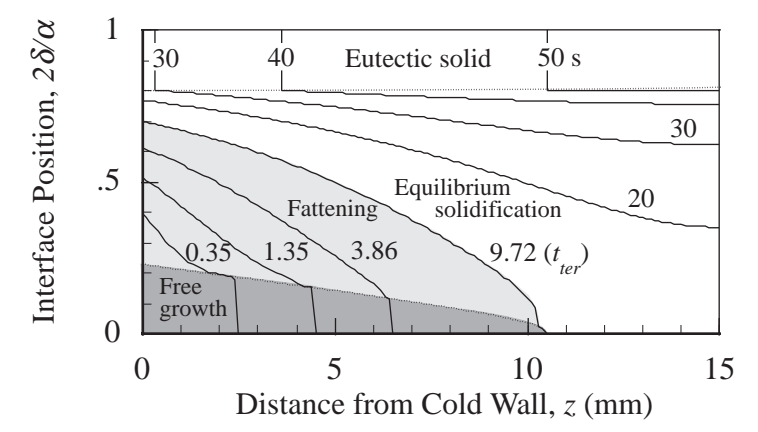

Fig. 4

.

.




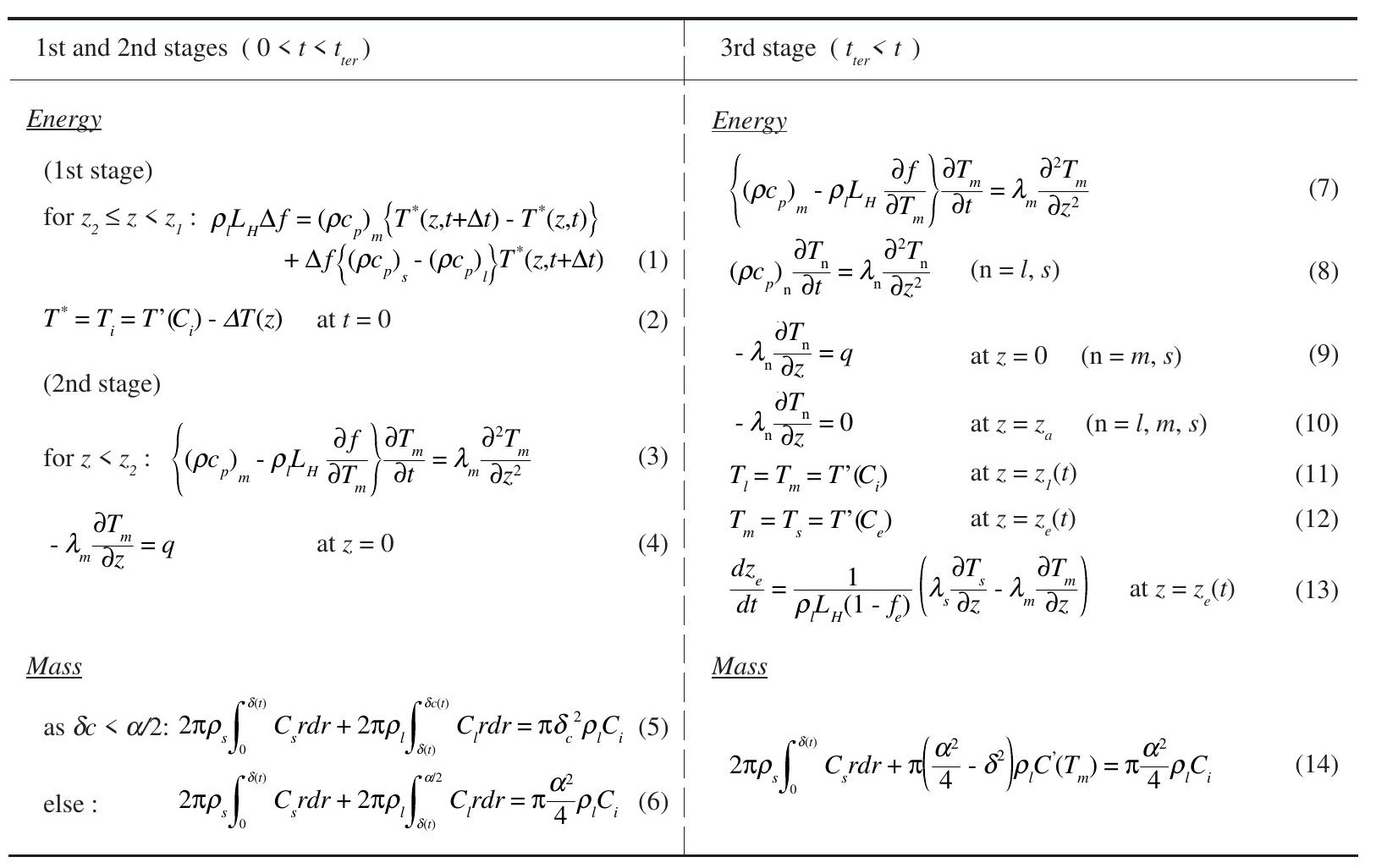

